\title{
Existence and multiplicity of solutions for a fractional $p$-Laplacian equation with perturbation
}

\author{
Zhen Zhi ${ }^{1,2}$, Lijun Yan $^{3}$ and Zuodong Yang ${ }^{4,5^{*}}$ (D)
}

\section{"Correspondence:}

zdyang_jin@263.net

${ }^{4}$ School of Teacher Education,

Nanjing Normal University, Jiangsu,

Nanjing 210097, China

${ }^{5}$ School of Teacher Education,

Nanjing University of Information

Science and Technology, Jiangsu,

Nanjing 210044, China

Full list of author information is

available at the end of the article

\begin{abstract}
In this paper, we consider the existence of nontrivial solutions for a fractional $p$-Laplacian equation in a bounded domain. Under different assumptions of nonlinearities, we give existence and multiplicity results respectively. Our approach is based on variational methods and some analytical techniques.
\end{abstract}

MSC: $35 J 20 ; 35 J 70$

Keywords: Multiplicity results; Fractional p-Laplacian; Critical points

\section{Introduction}

In this paper, we are interested in the existence of nontrivial solutions for the following fractional quasi-linear problem:

$$
\begin{cases}(-\Delta)_{p}^{s} u+|u|^{p-2} u=f(x, u)+\lambda g(x, u)+h(x), & x \in \Omega, \\ u=0, & x \in \mathbb{R}^{N} \backslash \Omega,\end{cases}
$$

where $0<s<1$ and $s p<N, \Omega$ is a bounded domain in $\mathbb{R}^{N}, \lambda$ is a positive constant, $f, g, h$ are continuous functions, $p_{s}^{*}=p N /(N-s p)$ is the fractional critical exponent, and $(-\Delta)_{p}^{s}$ is the fractional $p$-Laplace operator defined as

$$
(-\Delta)_{p}^{s} u(x)=\mathrm{P} . \mathrm{V} \cdot \int_{R^{N}} \frac{|u(x)-u(y)|^{p-2}(u(x)-u(y))}{|x-y|^{N+s p}} d y,
$$

where P.V. refers to the principle value, see [1] for details.

In recent years, there has been growing interest in the study of fractional elliptic equations. Concerning the existence result for this kind of equations, some well-known results for classical Laplace operators have been extended to the nonlocal fractional setting, and there are a lot of works on the quasi-linear problem

$$
\begin{cases}(-\Delta)_{p}^{s} u=f(x, u), & x \in \Omega, \\ u=0, & x \in \mathbb{R}^{N} \backslash \Omega,\end{cases}
$$

(c) The Author(s) 2021. This article is licensed under a Creative Commons Attribution 4.0 International License, which permits use, sharing, adaptation, distribution and reproduction in any medium or format, as long as you give appropriate credit to the original author(s) and the source, provide a link to the Creative Commons licence, and indicate if changes were made. The images or other third party material in this article are included in the article's Creative Commons licence, unless indicated otherwise in a credit line to the material. If material is not included in the article's Creative Commons licence and your intended use is not permitted by statutory regulation or exceeds the permitted use, you will need to obtain permission directly from the copyright holder. To view a copy of this licence, visit http://creativecommons.org/licenses/by/4.0/. 
where the nonlinearity $f$ satisfies some general growth conditions, see [2-12]. For instance, in $[2,4]$ the authors studied the fractional $p$-eigenvalue problems. In [5] the authors studied the local behavior of fractional $p$-minimizers. In [6] the authors studied problem (1.3) under different growth assumptions on the reaction term and obtained various existence results by Morse theory, while in [7] the authors studied problem (1.3) in an unbounded domain with weight, and symmetry results were given by authors in [8]. Moreover, by the variant fountain theorem the authors in [9] studied problem (1.3) in $\mathbb{R}^{N}$ and obtained infinitely many solutions, while similar results were obtained by authors in [3], given $f(x, u)=h_{1}(x)|u|^{q-2} u+h_{2}(x)|u|^{r-2} u$.

Typically, when $f(x, u)=|u|^{p_{s}^{*}-2} u+\lambda|u|^{p-2} u$, problem (1.3) turns into the BrezisNirenberg problem

$$
\begin{cases}(-\Delta)_{p}^{s} u=|u|^{p_{s}^{*}-2} u+\lambda|u|^{p-2} u, & x \in \Omega, \\ u=0, & x \in \mathbb{R}^{N} \backslash \Omega,\end{cases}
$$

which has been studied by the authors in [10-12]. In [10], the authors proved multiplicity results of problem (1.4) by cohomological index and abstract critical theorem, while in [11], the authors obtained nontrivial solutions of problem (1.4) by an abstract linking theorem. In [12], replacing $|u|^{p-2} u$ with a subcritical nonlinearity $g(x, u)$, the authors proved the existence of one weak solution of problem (1.4) provided $\lambda$ is sufficiently small, and multiplicity result was established if the perturbation term $g$ vanishes at the origin. Moreover, Kirchhoff type equations involving fractional $p$-Laplacian and critical nonlinearities were studied by authors in [13-15] by using variational methods.

Inspired by the above papers, we tend to investigate the existence and multiplicity result of problem (1.1). From our analysis, it is clear that under different assumptions of the growth condition on nonlinearities near infinity and origin, the existence and multiplicity results are quite different. We consider the Banach space $X=W_{0}^{s, p}(\Omega)$, where the fractional Sobolev space $W_{0}^{s, p}(\Omega)=\left\{u \in W^{s, p}(\Omega) \mid u=0 \in \mathbb{R}^{N} \backslash \Omega\right\}$ is defined as follows:

$$
W^{s, p}(\Omega)=\left\{u \in L^{p}(\Omega): \int_{\Omega \times \Omega} \frac{|u(x)-u(y)|^{p}}{|x-y|^{N+s p}} d x d y<\infty\right\},
$$

equipped with the norm

$$
\|u\|^{p}:=[u]_{s, p}^{p}=\iint_{Q} \frac{|u(x)-u(y)|^{p}}{|x-y|^{N+s p}} d x d y,
$$

where $Q=\mathbb{R}^{2 N} \backslash(C \Omega \times C \Omega)$ with $C \Omega=\mathbb{R}^{N} \backslash \Omega$. By the results of [1], there is continuous embedding $W_{0}^{s, p}(\Omega) \hookrightarrow L^{r}(\Omega)$ for $r \in\left[1, p_{s}^{*}\right]$ and compact when $r \in\left[1, p_{s}^{*}\right)$. We denote by $\mathrm{S}$ the best Sobolev constant for the embedding of $W_{0}^{s, p}(\Omega) \hookrightarrow L^{p}(\Omega)$.

Our approach to study problem (1.1) is variational, including the mountain pass theorem and the critical point theorems of G. Bonanno and R. Kajikiya. Generally, we check the geometric structure of the functional and prove the compactness results of the functional to meet the conditions of the critical point theorems. Due to the presence of critical nonlinearity, the energy functional no longer satisfies global compactness conditions but on certain ranges, thus we apply different variational theorems for existence results. We assume that the nonlinearities $f, g, h \in C(\mathbb{R}, \mathbb{R})$ and satisfy the following assumptions:

$\left(f_{1}\right) \lim _{t \rightarrow 0} \frac{f(x, t)}{t^{p-1}}=0$ uniformly a.e. $x \in \Omega$; 
$\left(\widetilde{f}_{1}\right) \lim _{t \rightarrow 0} \frac{f(x, t)}{t^{p-1}}=\infty$ uniformly a.e. $x \in \Omega$;

$\left(f_{2}\right) \lim _{t \rightarrow \infty} \frac{f(x, t)}{t^{*}-1}=0$ uniformly a.e. $x \in \Omega$;

$\left(\widetilde{f}_{2}\right) \lim _{t \rightarrow \infty} \frac{f(x, t)}{t^{p-1}}=0$ uniformly a.e. $x \in \Omega$;

$\left(f_{3}\right)$ there exists a positive constant $B$ such that

$$
F(x, s) \geq 2 r \frac{|s|^{p}}{p}-B, \quad \forall s \in \mathbb{R} \text {, a.e. } x \in \Omega,
$$

where $r$ is defined as $r:=\sup _{W^{s, p}(\Omega)} \frac{\|u\|^{p}}{|u|_{p}^{p}}$.

$\left(f_{4}\right)$ there exists $v \in\left(p, p_{s}^{*}\right)$ such that

$$
0<\nu F(t) \leq f(t) t \quad \text { for all }|t|>0 \text {, where } F(t):=\int_{0}^{t} f(s) d s
$$

$\left(g_{1}\right)|g(x, s)| \leq c\left(1+|s|^{r-1}\right), \forall s \in \mathbb{R}$, and $1 \leq r<p ;$

$\left(g_{2}\right) f(x, \cdot)$ is odd and $g(x, \cdot)$ is even for a.e. $x \in \Omega$;

$\left(g_{3}\right) f(x, \cdot)$ is odd and $g=|u|^{p^{*}-2} u$;

Our main results read as follows.

Theorem 1.1 Assume that $\left(f_{1}\right),\left(f_{2}\right),\left(f_{4}\right),\left(g_{1}\right)$ hold. Then there exists $\lambda^{*}>0$, and for any $\lambda \in$ $\left(0, \lambda^{*}\right)$, there exists $\varrho>0$ such that, for any $|f|_{\sigma}^{(p-1) / p} \in(0, \varrho)$, problem (1.1) has a mountain pass solution.

Theorem 1.2 Assume that $\left(f_{1}\right),\left(\widetilde{f}_{2}\right),\left(f_{3}\right),\left(g_{1}\right),\left(g_{2}\right)$ hold with $h=0$. Then, for every $b>0$, there exist an open interval $\Lambda \subset[-b, b]$ and a positive real number $\sigma$ such that, for every $\lambda \in \Lambda$, problem (1.1) admits at least three solutions whose norms are less than $\sigma$.

Theorem 1.3 Assume that $\left(\widetilde{f}_{1}\right),\left(f_{2}\right),\left(g_{3}\right)$ hold with $h=0$. Then there exists $\lambda_{*}$ such that for any $\lambda \in\left(0, \lambda_{*}\right)$, problem (1.1) has a sequence of nontrivial solutions $\left\{u_{n}\right\}_{n \in \mathbb{N}} \subset X$ such that $u_{n} \rightarrow 0$ as $n \rightarrow \infty$.

The present paper is organized as follows: in Sect. 2 we prove the existence of mountain pass solution, in Sect. 3 we prove the existence of three solutions, and in Sect. 4 we give infinitely many solutions for the critical case.

\section{Existence of mountain pass solution}

It is well known that the solution of problem (1.1) is a critical point of the functional $I$ : $X \rightarrow \mathbb{R}$ is defined by

$$
I(u)=\frac{1}{p}\|u\|^{p}-\lambda \int_{\Omega} G d x-\int_{\Omega} F d x-\int_{\Omega} h u d x
$$

and satisfies $\left\langle I^{\prime}(u), \varphi\right\rangle=0$, i.e.,

$$
\begin{aligned}
& \int_{Q} \frac{|u(x)-u(y)|^{p-2}(u(x)-u(y))}{|x-y|^{N+s p}}(\varphi(x)-\varphi(y))-\int_{\Omega} f(u) \varphi d x \\
& -\lambda \int_{\Omega} g(u) \varphi d x-\int_{\Omega} h \varphi d x=0
\end{aligned}
$$

for any $\varphi \in X$. 
We first check the mountain pass geometry of $I$.

Lemma 2.1 Assume that $\left(f_{1}\right),\left(f_{2}\right),\left(f_{4}\right),\left(g_{1}\right)$ hold, the functional I satisfies the mountain pass geometry:

(i) there exist $\alpha, \rho>0$ such that $I(u) \geq \alpha$ with $\|u\|=\rho$,

(ii) there exists $e \in X$ with $\|e\|>\rho$ such that $I(e)<0$.

Proof (i) By $\left(f_{1}\right)$ and $\left(f_{2}\right)$, for fixed $\varepsilon>0$, there exists $C_{\varepsilon}>0$ such that

$$
|F(t)| \leq \frac{\varepsilon}{p}|t|^{p}+\frac{C_{\varepsilon}}{p^{*}}|t|^{p^{*}}
$$

Taking into (2.1),

$$
\begin{aligned}
I(u) & =\frac{1}{p}\|u\|^{p}-\lambda \int_{\Omega} G d x-\int_{\Omega} F d x-\int_{\Omega} h u d x \\
& \geq \frac{1}{p}\|u\|^{p}-\lambda \int_{\Omega}\left(c|u|+\frac{c}{r}|u|^{r}\right) d x-\int_{\Omega}\left(\frac{\varepsilon}{p}|u|^{p}+C_{\varepsilon}|u|^{p^{*}}\right) d x-\int_{\Omega} h u d x \\
& \geq \frac{1}{2 p}\|u\|^{p}-\frac{c \lambda}{r \lambda_{1}}|\Omega|^{\frac{p-r}{p}}\|u\|^{r}-\frac{\lambda c}{\lambda_{1}}|\Omega|^{\frac{p-1}{p}}\|u\|-C_{\varepsilon} S\|u\|^{p^{*}}-C_{\varepsilon}^{\prime}|h|_{\sigma}^{\frac{p}{p-1}} \\
& =\|u\|^{p}\left[\frac{1}{2 p}-\lambda C_{1}\|u\|^{r-p}-\lambda C_{2}\|u\|^{1-p}-C_{3}\|u\|^{p^{*}-p}\right]-C_{\varepsilon}^{\prime}|h|_{\sigma}^{\frac{p}{p-1}} .
\end{aligned}
$$

Consider

$$
\zeta(t)=\lambda C_{1} t^{r-p}+\lambda C_{2} t^{1-p}+C_{3} t^{p^{*}-p} .
$$

It is easy to see $\lim _{t \rightarrow 0} \zeta(t)=\lim _{t \rightarrow+\infty} \zeta(t)=+\infty$. Let $\zeta^{\prime}(t)=0$, we have

$$
\lambda C_{1}(r-p) t^{r-p-1}+\lambda C_{2}(1-p) t^{-p}+C_{3}\left(p^{*}-p\right) t^{*^{*}-p-1}=0 .
$$

Note that $\zeta^{\prime}(t)=t^{-p} \eta(t)$, where

$$
\eta(t)=\lambda C_{1}(r-p) t^{r-1}+\lambda C_{2}(1-p)+C_{3}\left(p^{*}-p\right) t^{p^{*}-1} .
$$

Since $r<p<p^{*}$, there exists $t_{0}>0$ such that $\eta(t)<0$ on $\left(0, t_{0}\right)$ and $\eta(t)>0$ on $\left(t_{0}, \infty\right)$, i.e., $\zeta(t)$ has a unique minimum $\zeta\left(t_{0}\right)$, taking into, we have

$$
\zeta\left(t_{0}\right)=\lambda C_{3} t^{r-p}+\lambda C_{4} t^{1-p} .
$$

Thus there exists $\lambda^{*}>0$ such that, for $\lambda \in\left(0, \lambda^{*}\right), \zeta\left(t_{0}\right)<\frac{1}{4 p}$. Moreover, let

$$
\widetilde{\eta}\left(t_{1}\right)=\lambda C_{2}(1-p)+C_{3}\left(p^{*}-p\right) t_{1}^{p^{*}-1}=0
$$

it is easy to see that $t_{0}>t_{1}=\left[\frac{\lambda C_{2}(p-1)}{C_{3}\left(p^{*}-p\right)} \frac{1}{p^{*}-1}\right.$, thus there exists a constant $\varrho>0$ such that

$$
t_{0}^{p}\left[\frac{1}{2 p}-\zeta(t)\right]-C_{\varepsilon}^{\prime}|h|_{\sigma}^{\frac{p-1}{p}}>0
$$


for $|h|_{\sigma}^{\frac{p-1}{p}}<\varrho$, where $\varrho=\frac{\left[\frac{\lambda C_{2}(p-1)}{C_{3}\left(p^{*}-p\right)}\right]^{\frac{p}{p^{*}-1}}}{4 p C_{\varepsilon}^{\prime}}$. Thus, for $0<\lambda<\lambda^{*}$ and $|h|_{\sigma}^{\frac{p-1}{p}}<\varrho$, there exist $\alpha, \rho>$ 0 such that $I(u) \geq \alpha$ with $\|u\|=\rho$.

(ii) From $\left(f_{4}\right)$ we have that there exist $c_{1}, c_{2}>0$ such that

$$
F(s) \geq c_{1} s^{\nu}-c_{2} \quad \text { for all } s>0 .
$$

Thus, for any $u_{0}>0$ fixed, we have

$$
\begin{aligned}
I\left(t u_{0}\right) & =\frac{t^{p}}{p}\left\|u_{0}\right\|^{p}-\lambda \int_{\Omega} G\left(t u_{0}\right) d x-\int_{\Omega} F\left(t u_{0}\right) d x-t \int_{\Omega} h u_{0} d x \\
& \leq \frac{t^{p}}{p}\left\|u_{0}\right\|^{p}-c_{1} t^{\nu}\left|u_{0}\right|_{v}^{v}+c_{2}|\Omega|-\lambda \int_{\Omega} G\left(t u_{0}\right) d x-t \int_{\Omega} h u_{0} d x .
\end{aligned}
$$

Since $v \in\left(p, p^{*}\right)$, we get $I\left(t u_{0}\right) \rightarrow-\infty$ as $t \rightarrow \infty$. Thus there exists $e \in X$ with $\|e\|>\rho$ such that $I(e)<0$.

Lemma 2.2 Assume that $\left(f_{1}\right),\left(f_{2}\right),\left(f_{4}\right),\left(g_{1}\right)$ hold, then I satisfies the Palais-Smale condition.

Proof Suppose that $\left\{u_{n}\right\}_{n \in \mathbb{N}}$ is a Palais-Smale sequence of $I$, i.e., there exists $C>0$ such that

$$
I\left(u_{n}\right) \rightarrow C, \quad I^{\prime}\left(u_{n}\right) \rightarrow 0 \quad \text { as } n \rightarrow \infty,
$$

then we have

$$
\begin{aligned}
C & \left(1+\left\|u_{n}\right\|\right) \\
& \geq I\left(u_{n}\right)-\frac{1}{v}\left\langle I^{\prime}\left(u_{n}\right), u_{n}\right\rangle \\
& =\frac{1}{p}\left\|u_{n}\right\|^{p}-\lambda \int_{\Omega} G d x-\int_{\Omega} F d x-\int_{\Omega} h u_{n} d x-\frac{1}{v}\|u\|^{p}+\frac{1}{v} \int_{\Omega}(f+\lambda g+h) u_{n} d x \\
& =\left(\frac{1}{p}-\frac{1}{v}\right)\left\|u_{n}\right\|^{p}+\int_{\Omega}\left(\frac{f u_{n}}{v}-F\right) d x+\left(\frac{1}{v}-1\right) \int_{\Omega} h u_{n} d x+\lambda \int_{\Omega}\left(\frac{g u_{n}}{v}-G\right) d x \\
& \geq\left(\frac{1}{p}-\frac{1}{v}\right)\left\|u_{n}\right\|^{p}+\left(\frac{1-v}{v \lambda_{1}}\right)|h|_{\sigma}\left\|u_{n}\right\|-\frac{c^{\prime} \lambda}{r \lambda_{1}}|\Omega|^{\frac{p-r}{p}}\left\|u_{n}\right\|^{r}-\frac{\lambda c^{\prime}}{\lambda_{1}}|\Omega|^{\frac{p-1}{p}}\left\|u_{n}\right\| .
\end{aligned}
$$

Thus $\left\{u_{n}\right\}_{n \in \mathbb{N}}$ is bounded in $X$. Up to a subsequence, still denoted by $\left\{u_{n}\right\}_{n \in \mathbb{N}}$, there exists $u_{0} \in X$ satisfying

$$
u_{n} \rightarrow u_{0} \quad \text { in } W^{s, p}(\Omega), \quad u_{n} \rightarrow u_{0} \quad \text { in } L^{p}(\Omega), \quad u_{n}(x) \rightarrow u_{0}(x) \quad \text { a.e. on } \Omega .
$$

From $\left(f_{1}\right),\left(f_{2}\right),\left(g_{1}\right)$,we have by the Lebesgue convergence theorem

$$
\begin{aligned}
& \int_{\Omega} f\left(x, u_{n}\right)\left(u_{n}-u_{0}\right) d x \rightarrow 0, \quad \text { as } n \rightarrow \infty, \\
& \int_{\Omega} g\left(x, u_{n}\right)\left(u_{n}-u_{0}\right) d x \rightarrow 0, \quad \text { as } n \rightarrow \infty .
\end{aligned}
$$


Note that

$$
\begin{aligned}
\left\langle I^{\prime}\left(u_{n}\right), u_{n}-u_{0}\right\rangle= & \int_{\Omega} \frac{\left|u_{n}(x)-u_{n}(y)\right|^{p-2}\left(u_{n}(x)-u_{n}(y)\right)}{|x-y|^{N+s p}}\left(u_{n}(x)-u_{n}(y)-u_{0}(x)+u_{0}(y)\right) \\
& +\left|u_{n}\right|^{p-2} u_{n}\left(u_{n}-u_{0}\right)-\int_{\Omega} f\left(x, u_{n}\right)\left(u_{n}-u_{0}\right) d x \\
& -\lambda \int_{\Omega} g\left(x, u_{n}\right)\left(u_{n}-u_{0}\right)-\int_{\Omega} h(x)\left(u_{n}-u_{0}\right) d x,
\end{aligned}
$$

and

$$
\left\langle I^{\prime}\left(u_{n}\right), u_{n}-u_{0}\right\rangle \rightarrow 0 \quad \text { as } n \rightarrow \infty
$$

thus we have

$$
\begin{aligned}
& \int_{\Omega} \frac{\left|u_{n}(x)-u_{n}(y)\right|^{p-2}\left(u_{n}(x)-u_{n}(y)\right)}{|x-y|^{N+s p}}\left(u_{n}(x)-u_{n}(y)-u_{0}(x)+u_{0}(y)\right) \\
& \quad+\left|u_{n}\right|^{p-2} u_{n}\left(u_{n}-u_{0}\right) \rightarrow 0 .
\end{aligned}
$$

Combined with weak convergence of $u_{n} \rightarrow u_{0}$ in $W^{s, p}(\Omega)$, we have

$$
u_{n} \rightarrow u_{0} \quad \text { in } W^{1, p}(\Omega) \text { as } n \rightarrow \infty,
$$

thus I satisfies the Palais-Smale condition.

Proof of Theorem 1.1 In view of Lemma 2.1 and Lemma 2.2, Theorem 1.1 follows from the mountain pass theorem [16].

\section{Existence of three solutions}

In this section we consider multiplicity results of problem (1.1) when $h=0$,

$$
\begin{cases}(-\Delta)_{p}^{s} u+|u|^{p-2} u=f(x, u)+\lambda g(x, u) & x \in \Omega, \\ u=0, & x \in \mathbb{R}^{N} \backslash \Omega .\end{cases}
$$

We first recall the following theorem by G. Bonnano.

Lemma 3.1 ([17]) Let X be a separable and reflexive real Banach space, and let $\phi, \psi: X \rightarrow$ $\mathbb{R}$ be two continuously Gâteaux differentiable functionals. Assume that $\phi$ is sequentially weakly lower semicontinuous and even, that $\psi$ is sequentially weakly continuous and odd, and that, for some $b>0$ and for each $\lambda \in[-b, b]$, the functional $\psi+\lambda \phi$ satisfies the PalaisSmale condition and

$$
\lim _{\|x\| \rightarrow \infty}(\psi(x)+\lambda \phi(x))=+\infty
$$

Finally, assume that there exists $k>0$ such that

$$
\inf _{x \in X}(\psi(x))<\inf _{|\phi(x)|<k} \psi(x) .
$$


Then, for every $b>0$, there exist an open interval $\Lambda \subseteq[-b, b]$ and a positive real number $\sigma$ such that, for each $\lambda \in \Lambda$, the equation

$$
\psi^{\prime}(x)+\lambda \phi^{\prime}(x)=0
$$

admits at least three solutions in $X$ whose norms are less than $\sigma$.

Consider the functional

$$
I(u)=\frac{1}{p}\|u\|^{p}-\lambda \int_{\Omega} G d x-\int_{\Omega} F d x
$$

and denote $\psi(u)=\frac{1}{p}\|u\|^{p}-\int_{\Omega} F d x, \phi(u)=\int_{\Omega}-G d x$.

Proof of Theorem 1.2 It suffices to check that $I$ satisfies all the assumptions in Lemma 3.1. By $\left(\widetilde{f}_{2}\right)$, given $\epsilon>0$, we have

$$
\begin{aligned}
& |f(x, s)| \leq C_{\epsilon}+\epsilon|s|^{p-1} \quad \text { for every } s \in \mathbb{R}, \\
& |F(x, s)| \leq C_{\epsilon}+\frac{\epsilon}{p}|s|^{p} \quad \text { for every } s \in \mathbb{R}
\end{aligned}
$$

thus the functional $\psi(u)$ is continuously Gâteaux differentiable and weakly sequentially continuous. From $\left(g_{1}\right)$ we know that $\phi(u)$ is weakly sequentially continuous.

By $(3.5)$ and $\left(g_{1}\right)$, we derive

$$
\begin{aligned}
\psi(u)+\lambda \phi(u) & \geq \frac{1}{p}\|u\|^{p}-\int_{\Omega} C_{\epsilon}+\frac{\epsilon}{p}|s|^{p} d x-\lambda \int_{\Omega}\left(c|u|+\frac{1}{r}|u|^{r}\right) d x \\
& \geq \frac{1}{p}\left(1-\frac{\epsilon}{\lambda_{1}}\right)\|u\|^{p}-C_{1}\|u\|^{r}-C_{2}\|u\|-C_{3}|\Omega| .
\end{aligned}
$$

Since $p>r$, taking $\epsilon$ sufficiently small, we have

$$
\lim _{\|u\| \rightarrow+\infty} \psi(u)+\lambda \phi(u)=+\infty .
$$

Following a similar argument in Lemma 2.2, I satisfies the Palais-Smale condition.

By $\left(f_{1}\right)$, we have

$$
\int_{\Omega} F(x, u) d x \leq \frac{\epsilon}{p} \int_{\Omega}|u|^{p} d x \leq \frac{\epsilon}{p \lambda_{1}}\|u\|^{p} .
$$

Thus

$$
\psi(u) \geq \frac{1}{p}\|u\|^{p}-\frac{\epsilon}{p} \int_{\Omega}|u|^{p} d x>0 .
$$

Hence there exists $k>0$ such that

$$
\inf _{|\phi(u)|<k} \psi(u)=0 .
$$


Due to $\left(f_{3}\right)$, for any $u \in W^{s, p}(\Omega)$, let $t \rightarrow \infty$, there holds

$$
\begin{aligned}
\psi(t u) & =\frac{1}{p}\|t u\|^{p}-\int_{\Omega} F(x, t v) d x \\
& <\frac{t^{p}}{p}\|u\|^{p}-\frac{2 r t^{p}}{p} \int_{\Omega}|u|^{p} d x+B|\Omega| \\
& <-\frac{t^{p}}{p}+B|\Omega| \rightarrow-\infty .
\end{aligned}
$$

Then we have

$$
\inf _{u \in W^{s, p}(\Omega)} \psi(u)<\inf _{|\phi(u)|<k} \psi(u) .
$$

Thus completes the proof.

\section{Existence of infinitely many solutions}

In this section, we consider the critical case for problem (3.1), i.e.,

$$
\begin{cases}(-\Delta)_{p}^{s} u+|u|^{p-2} u=f(x, u)+\lambda|u|^{p_{s}^{*}-2} u, & x \in \Omega, \\ u=0, & x \in \mathbb{R}^{N} \backslash \Omega .\end{cases}
$$

Under assumption $\left(g_{3}\right)$ of Theorem 1.3, it is easy to see that the Euler-Lagrange functional of (4.1) is even, thus we tend to use the symmetric mountain pass theorem of Kajikiya for existence of infinitely many solutions. Due to the presence of critical term, we first prove the local compactness result.

Lemma 4.1 Let $\left(f_{2}\right)$ hold. Then, for any $M>0$, there exists $\lambda_{*}$ such that I satisfies the Palais-Smale condition on $(-\infty, M], \forall \lambda \in\left(0, \lambda_{*}\right)$.

Proof Let $\left\{u_{n}\right\}_{n \in \mathbb{N}}$ be a Palais-Smale sequence of $I$ at level $d$, i.e., there exists $d>0$ such that

$$
I\left(u_{n}\right) \rightarrow d, \quad I^{\prime}\left(u_{n}\right) \rightarrow 0 \quad \text { as } n \rightarrow \infty
$$

By $\left(f_{2}\right)$ we have

$$
|f(x, u) u| \leq c(\epsilon)+\epsilon|u|^{p_{s}^{*}}
$$

and

$$
|F(x, u)| \leq c^{\prime}(\epsilon)+\epsilon|u|^{p_{s}^{*}} .
$$

Then, by (4.2), we have

$$
d+o(1)\left\|u_{n}\right\|=I\left(u_{n}\right)-\frac{1}{p}\left\langle I^{\prime}\left(u_{n}\right), u_{n}\right\rangle
$$




$$
\begin{aligned}
& =\lambda\left(\frac{1}{p}-\frac{1}{p^{*}}\right)\left|u_{n}\right|_{p^{*}}^{p^{*}}+\int_{\Omega} \frac{1}{p} f u-F d x \\
& \geq\left(\frac{\lambda}{p}-\frac{\lambda}{p^{*}}-\epsilon\right)\left|u_{n}\right|_{p^{*}}^{p^{*}}-c(\epsilon)|\Omega| .
\end{aligned}
$$

Taking $\epsilon$ sufficiently small, we obtain

$$
\left|u_{n}\right|_{p^{*}}^{p^{*}} \leq C+o(1)\left\|u_{n}\right\|
$$

On the other hand,

$$
\begin{aligned}
d+o(1) & =\frac{1}{p}\left\|u_{n}\right\|^{p}-\frac{\lambda}{p^{*}}\left|u_{n}\right|_{p^{*}}^{p^{*}}-\int_{\Omega} F d x \\
& \geq \frac{1}{p}\left\|u_{n}\right\|^{p}-c^{\prime}(\epsilon)|\Omega|-\left(\frac{\lambda}{p^{*}}+\epsilon\right)\left|u_{n}\right|_{p^{*}}^{p^{*}}
\end{aligned}
$$

thus $\left\{u_{n}\right\}_{n \in \mathbb{N}}$ is bounded in $X$. Up to a subsequence, still denoted by $\left\{u_{n}\right\}_{n \in \mathbb{N}}$, there exists $u \in X$ satisfying

$$
u_{n} \rightarrow u \quad \text { in } W^{s, p}(\Omega), \quad u_{n} \rightarrow u \quad \text { in } L^{p}(\Omega), \quad u_{n}(x) \rightarrow u(x) \quad \text { a.e. on } \Omega \text {. }
$$

Applying $\left(f_{2}\right)$, we have

$$
\begin{aligned}
& \int_{\Omega} f\left(x, u_{n}\right) u_{n} d x=\int_{\Omega} f(x, u) u d x+o(1), \\
& \int_{\Omega} F\left(x, u_{n}\right) u_{n} d x=\int_{\Omega} F(x, u) u d x+o(1) .
\end{aligned}
$$

Noting that the sequence $\left\{\frac{\left|u_{n}(x)-u_{n}(y)\right|^{p-2}\left(u_{n}(x)-u_{n}(y)\right)}{|x-y|^{\frac{N+p s}{p}}}\right\}_{n \in \mathbb{N}}$ is bounded in $L^{p^{\prime}}(\Omega)$, by pointwise convergence $u_{n} \rightarrow u$, we have

$$
\frac{\left|u_{n}(x)-u_{n}(y)\right|^{p-2}\left(u_{n}(x)-u_{n}(y)\right)}{|x-y|^{\frac{N+p s}{p}}} \rightarrow^{L^{p^{\prime}}} \frac{|u(x)-u(y)|^{p-2}(u(x)-u(y))}{|x-y|^{\frac{N+p s}{p}}} .
$$

From (4.7)-(4.9) we derive $I^{\prime}(u)=0$, i.e., $\mathrm{u}$ is a weak solution of (4.1), and

$$
I(u)=\int_{\Omega}\left(\frac{1}{p} u f-F\right) d x+\lambda\left(\frac{1}{p}-\frac{1}{p^{*}}\right) \int_{\Omega}|u|^{p^{*}} d x .
$$

Thus it suffices to check $u_{n} \rightarrow u$ in $X$. We consider $v_{n}=u_{n}-u$. By the fractional form of the Brezis-Lieb lemma and (4.2), we have

$$
\begin{aligned}
\left\langle I^{\prime}\left(u_{n}, u_{n}\right\rangle\right. & =\left\|v_{n}\right\|^{p}+\|u\|^{p}-\int_{\Omega} u f(x, u) d x-\lambda \int_{\Omega}\left|v_{n}\right|^{p^{*}} d x-\lambda \int_{\Omega}|u|^{p^{*}} d x+o(1) \\
& =o(1)
\end{aligned}
$$

and

$$
I\left(u_{n}\right)=\frac{1}{p}\|u\|^{p}+\frac{1}{p}\left\|v_{n}\right\|^{p}-\int_{\Omega} F(x, u) d x-\frac{\lambda}{p^{*}} \int\left|v_{n}\right|^{p^{*}}-\frac{\lambda}{p^{*}} \int|u|^{p^{*}}+o(1) .
$$


Since $I^{\prime}(u)=0$, we have

$$
\left\|v_{n}\right\|^{p}=\lambda \int_{\Omega}\left|v_{n}\right|^{p^{*}} d x+o(1)
$$

Without loss of generality, we assume that $\left\|v_{n}\right\|^{p}=a+o(1)$. By the fractional Sobolev inequality, we have

$$
a \geq S(a / \lambda)^{p / p^{*}}
$$

If $a=0$, the proof is complete. Otherwise, $a \geq S^{N / p s} \lambda^{(p s-N) / p s}$. Combined with (4.2), (4.3), and (4.4), as $n \rightarrow \infty$ we derive

$$
\begin{aligned}
d & =\frac{a}{p}-\frac{a}{p^{*}}+\int_{\Omega}\left(\frac{1}{p} u f-F\right) d x+\lambda\left(\frac{1}{p}-\frac{1}{p^{*}}\right) \int_{\Omega}|u|^{p^{*}} d x \\
& \geq \frac{s}{n} S^{N / p s} \lambda^{(p s-N) / p s}-c^{\prime}(\epsilon)|\Omega|+\lambda\left(\frac{1}{p}-\frac{1}{p^{*}}-\epsilon\right) \int_{\Omega}|u|^{p^{*}} d x \\
& \geq \frac{s}{n} S^{N / p s} \lambda^{(p s-N) / p s}-c^{\prime}(s / 2 N \lambda)|\Omega|
\end{aligned}
$$

provided $\epsilon$ is sufficiently small. Then, given any $M>0$, there exists $\lambda_{*}$ such that

$$
d \geq \frac{s}{n} S^{N / p s} \lambda^{(p s-N) / p s}-c^{\prime}(\epsilon)>M
$$

for all $\lambda \in\left(0, \lambda_{*}\right)$, thus completes the proof.

Now we introduce Krasnoselski's genus. Let $E$ be a real Banach space. A closed subset $A$ of $E$ is called symmetric if $x \in A$ implies $-x \in A$. Denote by $\Sigma$ the family of all symmetric closed sets of $E$. The genus of $A$ is defined to be the smallest integer $\mathrm{n}$ if there is an odd map $\varphi \in C\left(A, \mathbb{R}^{n} \backslash\{0\}\right)$. If $\mathrm{n}$ does not exist, then $\gamma(A)=\infty$. Typically, $\gamma(\phi)=0$.

Proposition 4.2 Let $A, B \in \Sigma$. Then:

(1) If there exists an odd continuous map from $A$ to $B$, then $\gamma(A) \leq \gamma(B)$.

(2) If there is an odd homeomorphism from $A$ to $B$, then $\gamma(A)=\gamma(B)$.

(3) If $\gamma(B)<\infty$, then $\gamma(\overline{A \backslash B}) \geq \gamma(A)-\gamma(B)$.

(4) The $n$-dimensional sphere $\mathbb{S}^{n}$ has a genus of $n+1$ by the Borsuk-Ulam theorem.

(5) If $A$ is compact, then $\gamma(A)<\infty$, and there exist $\delta>0$ and a closed symmetric neighborhood $N_{\delta}(A)=\{x \in E:\|x-A\| \leq \delta\}$ of $A$ such that $\gamma\left(N_{\delta}(A)\right)=\gamma(A)$.

We then give the symmetric mountain pass lemma due to Kajikiya [18].

Lemma 4.3 Let $E$ be an infinite dimensional Banach space and $I \in C^{1}(E, \mathbb{R})$ be a functional satisfying the conditions below:

$\left(C_{1}\right) I(u)$ is even, bounded from below, $I(0)=0$, and $I(u)$ satisfies the local Palais-Smale condition, i.e., for some $d^{*}>0$, in the case when every sequence $\left\{u_{n}\right\}_{n \in \mathbb{R}^{N}}$ in E satisfying $I\left(u_{n}\right) \rightarrow d<d^{*}$ and $I^{\prime}\left(u_{n}\right) \rightarrow 0$ in $E^{*}$ has a convergent subsequence;

$\left(C_{2}\right)$ For each $n \in \mathbb{N}$, there exists $A_{n} \in \Gamma_{n}$ such that $\sup _{u \in A_{n}} I(u)<0$.

Then either $(i)$ or (ii) below holds.

(i) There exists a sequence $\left\{u_{n}\right\}_{n \in \mathbb{R}^{N}}$ such that $I^{\prime}\left(u_{n}\right)=0, I\left(u_{n}\right)=0$, and $\left\{u_{n}\right\}_{n \in \mathbb{R}^{N}}$ converges to 0 . 
(ii) There exist two sequences $\left\{u_{n}\right\}_{n \in \mathbb{R}^{N}}$ and $\left\{v_{n}\right\}_{n \in \mathbb{R}^{N}}$ such that $I^{\prime}\left(u_{n}\right)=0, I\left(v_{n}\right)<0$, $\lim _{n \rightarrow \infty} I\left(v_{n}\right)=0$, and $\left\{v_{n}\right\}_{n \in \mathbb{R}^{N}}$ converges to a nonzero limit.

Since $I(u)$ is not bounded from below, we use the truncation argument in the following discussion. Setting $\epsilon=\frac{\lambda}{p^{*}}$ in (4.4), it follows that

$$
\begin{aligned}
I(u) & =\frac{1}{p}\|u\|^{p}-\int_{\Omega} F d x-\frac{\lambda}{p^{*}} \int_{\Omega}|u|^{p^{*}} d x \\
& \geq \frac{1}{p}\|u\|^{p}-c\left(\frac{\lambda}{p *}\right)|\Omega|-\frac{2 \lambda}{p^{*}} \int_{\Omega}|u|^{p^{*}} d x \\
& \geq \frac{1}{p}\|u\|^{p}-c\left(\frac{\lambda}{p *}\right)|\Omega|-\frac{2 \lambda}{p^{*}} S^{-p^{* / p}}\|u\|^{p^{*}} \\
& =A\|u\|^{p}-B\|u\|^{p^{*}}-c\left(\frac{\lambda}{p *}\right)|\Omega|,
\end{aligned}
$$

where $A=\frac{1}{p}, B=\frac{2 \lambda}{p^{*}} S^{-p^{* / p}}, C=c\left(\frac{\lambda}{p^{*}}\right)|\Omega|$.

Consider

$$
g(t):=A t^{p}-B t^{p^{*}}-C
$$

it is easy to see that $g$ attains its maximum at $t_{1}=\left(\frac{S^{p^{*} / p}}{2 \lambda}\right)^{1 /\left(p^{*}-p\right)}$, and

$$
M_{1}=g\left(t_{1}\right)=\frac{s}{N}\left(\frac{S}{2}\right)^{N / p s} \lambda^{-n / p s}-c\left(\frac{\lambda}{p *}\right)|\Omega|>0
$$

provided $\lambda \in\left(0, \lambda_{*}^{\prime}\right)$, where $\lambda_{*}^{\prime}=\left[\frac{\frac{s}{N}\left(\frac{S}{2}\right)^{N / p s}}{C}\right]^{p s / N}$.

Thus we can find, for any $M_{0} \in\left(0, M_{1}\right), t_{0}<t_{1}$ such that $g\left(t_{0}\right)=M_{0}$. We then introduce the auxiliary function

$$
\chi(t)= \begin{cases}1, & 0 \leq t \leq t_{0}, \\ \frac{A t^{p}-C-M_{1}}{B t^{*}}, & t \geq t_{1}, \\ C^{\infty}, \chi(t) \in[0,1], & t_{0} \leq t \leq t_{1} .\end{cases}
$$

It is easy to see that $\chi(t) \in[0,1]$ and $\chi(t) \in C^{\infty}$. Let $\varphi(u):=\chi(\|u\|)$, and we consider the truncated functional $J: X \rightarrow \mathbb{R}$ defined as

$$
J(u)=\frac{1}{p}\|u\|^{p}-\varphi(u) \int_{\Omega} F d x-\frac{\lambda \varphi}{p^{*}} \int_{\Omega}|u|^{p^{*}} d x .
$$

Hence we have

$$
J(u) \geq A\|u\|^{p}-B \varphi(u)\|u\|^{p^{*}}-c\left(\frac{\lambda}{p *}\right)|\Omega|:=\bar{g}(\|u\|),
$$

where $\bar{g}(t)=A t^{p}-B \chi(t) t^{p^{*}}-C$ and

$$
\bar{g}(t):= \begin{cases}g(t), & 0 \leq t \leq t_{0} \\ M_{1} & t \geq t_{1}\end{cases}
$$


By the above arguments, we have the following result.

Lemma 4.4 Let $J(u)$ be defined as in (4.17), then

(i) $J \in C^{1}(X, \mathbb{R}), J$ is even and bounded from below.

(ii) If $J(u)<M_{0}$, then $\bar{g}(\|u\|)<M_{0}$, and consequently $\|u\|<t_{0}$ with $I(u)=J(u)$.

(iii) There exists $\lambda_{*}$ such that, for any $\lambda \in\left(0, \lambda_{*}\right)$, $J$ satisfies a local Palais-Smale condition for

$$
d<M_{0} \in\left(0, \min \left\{M_{1}, \frac{s}{n} S^{N / p s} \lambda^{(p s-N) / p s}-c^{\prime}(s / 2 \lambda N)\right\}\right)
$$

Proof It is easy to see (i) and (ii). (iii) holds consequently by (ii) and Lemma 4.1.

Lemma 4.5 Assume that $\left(\widetilde{f}_{1}\right)$ holds. Then, for any $k \in \mathbb{N}$, there exists $\delta(k)>0$ such that $\gamma(\{u \in X: J(u) \leq \delta(k)\} \backslash\{0\}) \geq k$.

Proof By $\left(\tilde{f}_{1}\right)$, we derive

$$
F(x, \varepsilon u) \geq G(\varepsilon)(\varepsilon u)^{p} \quad \text { with } G(\varepsilon) \rightarrow \infty \text { as } \varepsilon \rightarrow 0 .
$$

Given $k \in \mathbb{N}$ and let $E_{k}$ be a k-dimensional subspace of $X$. Since all norms in $E_{k}$ are equivalent, we define

$$
\alpha_{k}=\inf _{\|u\|=1} \int_{\Omega}|u|^{p^{*}} d x, \quad \beta_{k}=\inf _{\|u\|=1} \int_{\Omega}|u|^{p} d x,
$$

then for any $u \in E_{k}$ and $\varepsilon \in\left(0, t_{0}\right)$,

$$
\begin{aligned}
I(\varepsilon u) & =J(\varepsilon u) \\
& \leq \frac{\varepsilon^{p}}{p}-\frac{\lambda \varepsilon^{p^{*}}}{p^{*}} \alpha_{k}-G(\varepsilon) \varepsilon^{p} \beta_{k} \\
& \leq \varepsilon^{p}\left[\frac{1}{p}-\frac{\lambda \varepsilon^{p^{*}-p}}{p^{*}} \alpha_{k}-G(\varepsilon) \beta_{k}\right] \\
& =-\delta(k)<0
\end{aligned}
$$

provided $\varepsilon$ is sufficiently small, since $G(\varepsilon) \rightarrow \infty(\varepsilon \rightarrow 0)$. Thus

$$
\left\{u \in E_{k}:\|u\|=\varepsilon\right\} \subset\{u \in X: J(u) \leq-\delta(k)\} \backslash\{0\} .
$$

This completes the proof.

Proof of Theorem 1.3 Consider

$$
\Sigma_{k}:=\{A \in X \backslash\{0\}: A \text { is closed and } A=-A, \gamma(A) \geq k\} \text {, }
$$

and define

$$
c_{k}=\inf _{A \in \Sigma_{k}} \sup _{u \in A} G(u) .
$$


By Lemma 4.4-(i) and Lemma 4.5, it implies $-\infty<c_{k}<0$. Thus conditions $\left(C_{1}\right)$ and $\left(C_{2}\right)$ of Lemma 4.3 are satisfied. Consequently, there exists a sequence of solutions $\left\{u_{n}\right\}$ converging to 0 . Therefore, Theorem 1.3 follows by Lemma 4.4(ii).

\section{Funding}

Project Supported by the National Natural Science Foundation of China (Grant No. 11571093); the Natural Science Foundation of Jiangsu Education Commission China (Grant No. 19KJB110016); the Fundamental Research Funds for the Central Universities of China (Grant No. 3142020023); and the Science and Technology Support Project of Langfang (No. 2020011016)

\section{Availability of data and materials}

Data sharing not applicable to this article as no data sets were generated or analyzed during the current study.

\section{Competing interests}

The authors declare that they have no competing interests.

\section{Authors' contributions}

Each of the authors contributed to each part of the work equally, all authors read and approved the final manuscript.

\section{Author details}

'School of Computer Science and Artificial Intelligence \& Aliyun School of Big Data, Changzhou University, Jiangsu, Changzhou 213164, China. ${ }^{2}$ Institute of Mathematics, School of Mathematics Science, Nanjing Normal University, Jiangsu, Nanjing 210023, China. ${ }^{3}$ School of Science, North China Institute of Science and Technology, Hebei, Sanhe 065201, China. ${ }^{4}$ School of Teacher Education, Nanjing Normal University, Jiangsu, Nanjing 210097, China. ${ }^{5}$ School of Teacher Education, Nanjing University of Information Science and Technology, Jiangsu, Nanjing 210044, China.

\section{Publisher's Note}

Springer Nature remains neutral with regard to jurisdictional claims in published maps and institutional affiliations.

Received: 24 December 2020 Accepted: 21 May 2021 Published online: 01 June 2021

\section{References}

1. Di Nezza, E., Palatucci, G., Valdinoci, E.: Hitchhiker's guide to the fractional Sobolev spaces. Bull. Sci. Math. 136, 521-573 (2012)

2. Franzina, G., Palatucci, G.: Fractional p-eigenvalues. Riv. Mat. Univ. Parma 5(1), 373-386 (2014)

3. Chen, Q., Chen, C., Shi, Y.: Multiple solutions for fractional $p$-Laplace equation with concave-convex nonlinearities. Bound. Value Probl. 2020, 63 (2020)

4. Lindgren, E., Lindqvist, P.: Fractional eigenvalues. Calc. Var. 49, 795-826 (2014)

5. Castro, A., Kuusi, T., Palatucci, G.: Local behavior of fractional p-minimizers. Ann. Inst. Henri Poincaré, Anal. Non Linéaire 33(5), 1279-1299 (2016)

6. Iannizzotto, A., Liu, S., Perera, K., Squassina, M.: Existence results for fractional $p$-Laplacian problems via Morse theory. Adv. Calc. Var. 9(2), 101-125 (2016)

7. Lehrer, R., Maia, L., Squassina, M.: On fractional p-Laplacian problems with weight. Differ. Integral Equ. 28, 15-28 (2015)

8. Ledesma, C.: Existence and symmetry result for fractional $p$-Laplacian in $\mathbb{R}^{N}$. Commun. Pure Appl. Anal. 16, 99-113 (2017)

9. Ambrosio, V.: Multiple solutions for a fractional $p$-Laplacian equation with sign-changing potential. Electron. J. Differ. Equ. 2016, 151 (2016)

10. Perera, K., Squassina, M., Yang, Y.: Bifurcation and multiplicity results for critical fractional $p$-Laplacian problems. Math. Nachr. 289, 332-342 (2015)

11. Mosconi, S., Perera, K., Squassina, M., Yang, Y.: The Brezis-Nirenberg problem for the fractional p-Laplacian. Calc. Var. Partial Differ. Equ. 55, 55-105 (2016)

12. Mawhin, J., Bisci, G.: A Brezis-Nireberg type result for a nonlocal fractional operator. J. Lond. Math. Soc. 95(2), 73-93 (2017)

13. Pucci, P., Xiang, M., Zhang, B.: Multiple solutions for nonhomogeneous Schrödinger-Kirchhoff type equations involving the fractional $p$-Laplacian in $\mathbb{R}^{N}$. Calc. Var. 54, 2785-2806 (2015)

14. Caponi, M., Pucci, P.: Existence theorems for entire solutions of stationary Kirchhoff fractional $p$-Laplacian equations. Ann. Math. 195, 2099-2199 (2016)

15. Liang, S., Pu, H., Rădulescu, V.: High perturbations of critical fractional Kirchhoff equations with logarithmic nonlinearity. Appl. Math. Lett. 116, 107027 (2021)

16. Rabinowitz, P.H.: Minimax Methods in Critical Point Theory with Applications to Differential Equations. Am. Math. Soc., Providence (1986)

17. Bonanno, G.: Some remarks on a three critical points theorem. Nonlinear Anal. 54, 651-665 (2003)

18. Kajikiya, R.: A critical-point theorem related to the symmetric mountain-pass lemma and its applications to elliptic equations. J. Funct. Anal. 225, 352-370 (2005) 